\title{
Métodos semiempíricos de química quântica no ensino da entalpia das reações químicas
}

Semiempirical quantum chemistry methods in teaching the enthalpy of chemical reactions

\author{
L. S. Martins ${ }^{1}$; A. H. Lima ${ }^{2 *}$ \\ ${ }^{1}$ Curso de Ciências Naturais/Universidade do Estado do Pará(UEPA), 68447-000, Barcarena-PA, Brasil \\ ${ }^{2}$ Insitituto de Ciências Exatas e Naturais / Laboratório de Planejamento e Desenvolvimento de Fármacos, \\ Universidade Federal do Pará, CEP 66075-110, Belém-PA, Brasil \\ *anderson@ufpa.br
}

(Recebido em 22 de março de 2016; aceito em 26 de abril de 2016)

\begin{abstract}
A carência de métodos para auxiliar o ensino de Química torna o assunto desafiador diante das didáticas de ensino. No entanto, pesquisas têm sido feitas para propor novas metodologias que visam contribuir neste sentido. Dentre as propostas, o uso do computador no auxílio ao aprendizado se destaca como sendo uma das mais promissoras. Essa metodologia pode ser bem-vinda no tratamento didático da entalpia, pois, entender esse significado requer conhecer o problema que lhe deu origem e o encaminhamento da sua solução. Neste sentido, foram realizadas simulações em um software de análise química teórica, a fim de obter a entalpia de algumas reações e compará-las com as suas entalpias experimentais. Além disso, fezse a aplicação desse método junto a graduandos em química para verificar a viabilidade em aplicar esta atividade. Assim, este trabalho apresenta a Química computacional através de métodos quânticos semiempíricos como complemento ao ensino de entalpia das reações Químicas na graduação.

Palavras-chave: Química computacional, Entalpia, Ensino de Química
\end{abstract}

The lack of methods to assist the chemical teaching makes the challenging issue facing the teaching didactics. However, research has been done to propose new methodologies that aim to contribute to this. Among the proposals, the use of computers to aid the learning stands out as one of the most promising. This methodology may be useful in the didactic treatment of enthalpy therefore understand this significance requires knowing the problem that gave rise to and its solutions, as well. In this sense, simulations were performed on a theoretical chemical analysis software in order to obtain the enthalpy of some reactions and comparing them with their experimental enthalpy. Besides that, application of this method with chemistry students has been employed in order to verify the viability of this activity. Thus, this work presents the computational chemistry using quantum semiempirical methods as a complement to the enthalpy of teaching of chemical reactions for undergraduate students.

Keywords: Computational Chemistry, Enthalpy, Chemistry teaching.

\section{INTRODUÇÃO}

Nos dias atuais torna-se evidente a percepção de uma grande demanda de informações que são direcionadas aos alunos, mas nem sempre são tratadas adequadamente. Nesse contexto, uma ampla parte desta informação é analisada pelo professor que, no avanço da concepção técnicocientífica, cada vez mais tem que elaborar habilidades em seus alunos, o que necessita em muitos casos, um trabalho extenso e contextualizado [1].

A definição e assimilação dos conteúdos de Química é um processo complexo no qual provém de variados fatores [2]. Por esse motivo, a ausência de recursos para auxiliar a docência de certos tópicos do conteúdo de Química faz com que, o assunto se torne carente em relação às didáticas de ensino, dificultando o processo de ensino-aprendizagem.

Singularmente no ensino de Química, percebe-se que o aluno não consegue associar o conteúdo com as possibilidades e ferramentas do seu cotidiano [1]. Nesse segmento, aprimorar o entendimento dos alunos sobre os conceitos de Química tem sido um dos objetivos centrais dos professores acerca da pesquisa e do ensino dessa ciência. Dentre as várias metodologias propostas, a utilização do computador como meio de auxílio ao aprendizado se destaca como sendo uma das mais promissoras e satisfatórias [3]. Desde então, a aliança feita entre Ciência e 
Tecnologia provocou grandes mudanças que possibilitaram o crescimento tanto de uma, quanto de outra [4].

Dentro dos tópicos que a Química dispõe, as maiores dificuldades apontadas e enfrentadas pelos alunos estão presentes nos conceitos da Termodinâmica Química, em que artigos científicos citam a dificuldade em assimilar conceitos fundamentais como: calor, temperatura, energia interna, entalpia, entropia, energia cinética e potencial associada a átomos e moléculas [5-8]. Devido a essas dificuldades apontadas no ensino destas concepções, argumenta-se que, para a plena compreensão de conceitos, como entalpia, seria primordial um aprofundamento nas ideias científicas a nível superior [8].

A fim de propor um novo norte e amenizar a falta de diversificação no ensino de entalpia das reações químicas, o presente trabalho propõe o uso da química computacional como método de auxílio para o ensino desse conteúdo na graduação. Para isso, foram realizados por meio de um software de química computacional (MOPAC) [9], cálculos utilizando métodos semiempíricos da química teórica, a fim de obter a entalpia de algumas reações químicas de combustão e compará-las com as suas respectivas entalpias de reações dadas experimentalmente. Além disso, objetivou-se aplicar essa atividade junto a alunos graduandos do curso de Licenciatura em ciências naturais com habilitação em química com o intuito de verificar a viabilidade do uso destes procedimentos no ensino de química na graduação. No entanto, alguns conceitos e abordagens específicas da química teórica necessitaram ser devidamente compreendidas para que possam ser aplicadas de maneira eficaz. Com esta proposta, espera-se diversificar as técnicas de ensino de entalpia das reações químicas, ampliando a participação dos universitários no estudo do tema.

\subsection{Princípios da Termodinâmica e Entalpia}

O progresso da termodinâmica como campo de estudo, começou por volta do século XVII. $\mathrm{Na}$ ocasião o químico e físico Robert Boyle, realizava trabalhos experimentais comprovando as ligações macroscópicas presente entre temperatura, volume e pressão nas substâncias gasosas. As leis inferidas desde então com relação a esses experimentos, esclareceram o comportamento de sistemas físicos macroscópicos, desatrelado, na sua maioria, de quaisquer hipóteses relacionadas com o caráter atômico-molecular da matéria [10-12]. Contudo, algumas questões dos aspectos atômico-molecular nos proporcionam um melhor entendimento ao tratar a dinâmica dos processos de transferência de energia [13]. E essa concepção, é descendência de conceitos mais profundos, unificando vários conhecimentos dentre as ciências [14].

A termodinâmica, que é um ramo da física que estuda os processos de transferência de energia, se fundamenta basicamente em duas leis naturais para seus estudos. A primeira lei, analisa e acompanha as variações de energia de um sistema e proporciona o cálculo da quantidade de calor que uma reação absorve ou libera. A variação da energia interna $(\Delta U)$ de um sistema também é observada pela primeira lei da termodinâmica, a qual representa a aplicação do princípio de conservação da energia a sistemas que podem trocar energia com a vizinhança por calor e por trabalho. Se um sistema troca energia com a vizinhança por trabalho e por calor, então a variação da sua energia interna é dada por [15]:

$$
\Delta U=q+w
$$

A segunda lei analisa a espontaneidade das transformações e explica por que algumas reações ocorrem espontaneamente, enquanto outras não. A espontaneidade de uma reação tende a crescer com o aumento da entropia (S), a qual é tratada como uma medida da maior dispersão de energia de um sistema (ou sistema mais vizinhanças) [16]. A variação de entropia de um sistema pode ser calculada pela equação 2 .

$$
\Delta S=\frac{q_{r e v}}{T}
$$


Em que $q$ é o calor transferido e T é a temperatura absoluta na qual ocorre a transferência. $\mathrm{O}$ termo rev indica que o calor transferido é reversível de acordo com os princípios da termodinâmica [15].

Uma consequência da primeira lei da termodinâmica é o caso específico da entalpia $(\mathrm{H})$, que é definida como:

$$
H=U+P V
$$

Em que $\mathrm{U}, \mathrm{P}$ e $\mathrm{V}$ são a energia interna, a pressão e o volume do sistema, respectivamente. A consequência principal dessa definição junto à primeira lei é que, a variação de entalpia de um sistema é igual ao calor envolvido na transformação a pressão constante. Como pode ser observado na equação 4 , a energia interna de um sistema varia junto com seu volume [17].

$$
\Delta H=\Delta U+P \Delta V
$$

Então, usando a primeira lei da termodinâmica e julgando que o sistema só pode realizar trabalho de expansão, que é o trabalho realizado contra uma pressão externa $\left(-\mathrm{P}_{e x t}\right)$, temos:

$$
\begin{gathered}
\Delta H=q+w+P \Delta V \\
\Delta H=q-P_{e x t} \Delta V+P \Delta V
\end{gathered}
$$

Assim sendo, com o sistema aberto para a atmosfera a pressão é igual à pressão externa. Resultando em:

$$
\Delta H=q
$$

Pode-se aplicar esse mesmo princípio as variações de energia que acompanham uma reação química, chamada de entalpia de reação [15]. A variação de energia do sistema presente em uma reação química, a pressão constante, é estabelecida por $\Delta_{r} H^{\theta}$, que é determinada sendo a diferença entre o somatório das entalpias molares dos produtos, e o somatório das entalpias molares dos reagentes, acompanhadas pelas suas respectivas estequiometrias [17], de acordo com a equação 8 .

$$
\Delta_{r} H^{\theta}=\sum_{\text {produtos }} v H_{m}^{\theta}-\sum_{\text {reagentes }} v H_{m}^{\theta}
$$

Na qual, $H_{m}^{\theta}$ corresponde à entalpia molar padrão da substância selecionada, em uma dada temperatura e $v$ é o coeficiente estequiométrico da substância.

\subsection{Métodos semiempíricos de química quântica}

Em química computacional, métodos semiempíricos ocupam uma posição intermediária entre a mecânica molecular e métodos $a b$ initio [18]. Os métodos semiempíricos contém mais restrições matemáticas que os métodos $a b$ initio, mas que são corrigidas por meio do uso de parâmetros ajustáveis, que proporcionam a redução do custo computacional [19]. Este fator diminui o tempo computacional dos cálculos em química teórica ao mesmo tempo em que gera resultados relativamente satisfatórios de modo que grandes moléculas podem ser modeladas de maneira realista $[18,20]$.

A decisão de escolha de um método computacional para a solução de um problema químico não é uma tarefa fácil. A exatidão relacionada a estes métodos e o custo do cálculo em termos de velocidade e memória computacional tem que ser analisada [21]. Hoje em dia, muitos métodos semiempíricos são utilizados em química teórica como o método Austin Model 1 (AM1) [22], Parametric Method 3 (PM3) [23] e Recife Model 1 (RM1) [24], que são métodos semiempíricos que usam aproximações semelhantes, mas possuem diferenças nas suas parametrizações. Além destes, há ainda os métodos Parametric Method 6 (PM6) [25] Parametric 
Method 7 (PM7) [26] entre outros. Vale ressaltar que cada método realiza a escolha de quais parâmetros serão ajustados, mas o princípio geral é o mesmo [21].

\subsection{Uso de Software na Educação}

Os profissionais que atuam na área da educação vêm vivenciando a inserção das redes tecnológicas na sua rotina diária. Essa inclusão faz com que o computador se torne uma ferramenta dinâmica em sistemas de educação. As tendências de uso do computador na educação já mostram que ele pode ser um aliado neste processo que estamos começando a entender [27].

Os aparatos tecnológicos não devem entrar em sala de aula ou no ambiente de ensino, sem que os profissionais atuantes na mesma, principalmente os docentes, estejam conscientes que a tecnologia irá contribuir significativamente para o progresso das atividades junto ao corpo discente, ajudando a promover melhor os objetivos educacionais que consideram valiosos [28].

Todavia, é necessário comentar que existem variadas formas de classificar os softwares que são utilizados na educação. Por exemplo, softwares educativos podem ser classificados em tutor (o software que instrui o aluno), tutorado (software que permite o aluno instruir o computador) e ferramenta (software com o qual o aluno manipula a informação) [29].

$\mathrm{O}$ uso do cenário virtual proporciona a simulação de situações difíceis, arriscadas ou custosas, o que possibilita ao aluno ter a chance de trabalhar o conhecimento que seria puramente teórico usando um ambiente realístico [30]. A aplicação de softwares químicos passa para os computadores a complicada missão de realizar os cálculos, que seriam impossíveis de dar-se manualmente, proporcionando ao aluno apenas raciocinar a respeito das hipóteses assumidas, na interpretação das soluções, no contexto de validade dos modelos e nas possíveis generalizações/expansões do modelo que possam ser realizadas [31].

Com a evolução do poder computacional, muitos softwares que eram utilizados como coadjuvantes na pesquisa científica, hoje desempenham papéis fundamentais auxiliando no entendimento de muitos conteúdos, como, reações enzimáticas, modelagem de proteínas, etc. Aliado a isso, os resultados que estas pesquisas trazem estão sendo cada vez mais aplicados no ensino de propriedades químicas importantes que poderão levar o aluno a um melhor entendimento da problemática em questão [32,33].

\section{METODOLOGIA}

\subsection{Cálculos no programa MOPAC 2012}

Inicialmente foram selecionadas algumas reações de combustão (apresentadas na Figura 1), as quais pretendia-se obter seus valores de entalpia a partir de métodos da Química Teórica Computacional.

$\begin{array}{ccccccc}\mathrm{CH}_{4} & + & 2 \mathrm{O}_{2} & \rightarrow & \mathrm{CO}_{2} & + & 2 \mathrm{H}_{2} \mathrm{O} \\ \mathrm{C}_{3} \mathrm{H}_{8} & + & 5 \mathrm{O}_{2} & \rightarrow & 3 \mathrm{CO}_{2} & + & 4 \mathrm{H}_{2} \mathrm{O} \\ \mathrm{C}_{4} \mathrm{H}_{10} & + & 13 / 2 \mathrm{O}_{2} & \rightarrow & 4 \mathrm{CO}_{2} & + & 5 \mathrm{H}_{2} \mathrm{O} \\ \mathrm{C}_{2} \mathrm{H}_{5} \mathrm{OH} & + & 3 \mathrm{O}_{2} & \rightarrow & 2 \mathrm{CO}_{2} & + & 3 \mathrm{H}_{2} \mathrm{O} \\ \mathrm{CH}_{3} \mathrm{OH} & + & 3 / 2 \mathrm{O}_{2} & \rightarrow & \mathrm{CO}_{2} & + & 2 \mathrm{H}_{2} \mathrm{O}\end{array}$

Figura 1. Reações realizadas no trabalho

Primeiramente, desenhou-se no programa MarvinSketch (versão 5.0, ChemAxon, http://www.chemaxon.com), todas as moléculas envolvidas nas reações selecionadas. Em 
seguida, para realização dos cálculos da entalpia das reações foi utilizado o programa MOPAC2012 [9].

Os arquivos de entrada (inputs) foram preparados baseados nas palavras-chave disponível no manual do programa (http://openmopac.net/manual/index.html). Assim, a primeira linha do arquivo de entrada apresenta a seguinte forma: "AM1 OPT GNORM=0.01 PRECISE XYZ CHARGE $=0$ ". Onde, "AM1" especifica que o método semiempírico AM1 foi utilizado no cálculo. No caso deste trabalho, os cálculos foram repetidos utilizando os métodos RM1, PM6 e PM7. OPT, permite otimizar as coordenadas de todos os átomos, neste caso, as geometrias foram otimizadas utilizando a rotina "eigenvector following (EF)" a qual é a padrão no MOPAC2012. GNORM=0.01, permite parar a otimização da geometria quando o gradiente se torna menor do que $0.01 \mathrm{kcal} / \AA$. PRECISE, trata de que os critérios para o encerramento de todas as otimizações devem ser aumentados por um fator, normalmente 100. PRECISE pode ser usado quando resultados mais precisos são desejados. XYZ, especifica que todos os átomos serão convertidos em coordenadas cartesianas e o cálculo será executado inteiramente em coordenadas cartesianas. Finalmente, $\mathrm{CHARGE}=0$ indica que a carga do sistema é zero. Este valor deve ser alterado de acordo com o sistema trabalhado. Além disso, é conveniente expressar o estado de spin das moléculas em questão. Isto pode ser feito adicionando as palavras-chave singlet, triplet, quartet, etc. Como exemplo, neste trabalho utilizou-se o $\mathrm{O}_{2}$ triplet, que é a forma energeticamente mais favorável da molécula de oxigênio.

A partir da fórmula da variação da entalpia (equação 8) pôde-se calcular a entalpia das reações desejadas. As entalpias calculadas foram comparadas com seus respectivos valores experimentais a fim de verificar qual dos métodos semiempíricos apresentava maior correlação.

Para a realização dos cálculos químico-quânticos, foi utilizado um computador considerado intermediário quanto ao seu desempenho HD320GB, memória 2GB DDR3, processador Intel N455 e sistema operacional Windows 7 Starter.

\subsection{Aplicação com os Graduandos}

A atividade foi aplicada junto a 12 alunos graduandos do curso de licenciatura em ciências naturais com habilitação em Química da Universidade do Estado do Pará (UEPA), no laboratório de informática do campus, com duração de duas horas.

Nos dias que antecederam a atividade, foi aplicado um questionário aos alunos com questões relacionadas à termodinâmica química com ênfase em entalpia e também química computacional a fim de obter informações acerca dos conhecimentos prévios dos alunos.

A partir do resultado dos questionários pôde-se elaborar a primeira parte do treinamento, que se tratou de uma aula teórica sobre aspectos da termoquímica e alguns conteúdos relevantes para a aplicação e compreensão dos métodos. Esta etapa teve duração de quarenta minutos e iniciouse momentos antes do treinamento.

Depois da aula teórica deu-se início a aplicação do método em que, foi distribuído aos alunos um roteiro da atividade proposta. Ao término das atividades foi pedido aos graduandos que avaliassem o método que foi aplicado tendo em vista os aspectos positivos e negativos para sua inserção no ensino de Química dentro da graduação.

\section{RESULTADOS E DISCUSSÃO}

Em geral nos cálculos de Química computacional, percebe-se a necessidade de conhecimentos desenvolvidos de informática, além disso, em sua maior parte, os procedimentos são bastante específicos no conteúdo a ser desenvolvido com os métodos computacionais e os princípios da química quântica utilizados. Porém, no presente trabalho, os cálculos de simulação sugeridos pelo autor foram realizados de forma a fazer com que a atenção fosse voltada para a Química envolvida e não com a bagagem de teorias envolvidas na Química computacional.

O software MOPAC2012 utiliza princípios quânticos e termodinâmicos, além de um pouco de matemática bastante avançada. No entanto, o usuário não precisa estar proficiente com estes tópicos especializados. Os comandos de entrada são conservados o mais simples possível, de 
uma maneira que permite aos usuários dar a sua atenção para a Química envolvida e não se atentar muito com as teorias quânticas e a exótica termodinâmica [9]. O MOPAC2012 atualmente é mais utilizado como ferramenta de pesquisa, mas por esses fatores didáticos pretende-se usar o mesmo como um complemento na didática do ensino da termodinâmica química.

Neste trabalho, a escolha das reações de combustão para os cálculos das entalpias de formação se deu pelo fato destas reações serem comuns no cotidiano dos alunos. Além disso, optou-se por compostos que possuíssem o menor número de átomos envolvidos no processo. Isso se dá a fim de otimizar o tempo de simulação computacional.

\subsection{Cálculos no MOPAC e valores experimentais}

Os arquivos de saída (output) deste software não fornecem diretamente uma estimativa para o valor da variação de entalpia da reação, mas fornece diretamente a estimativa da entalpia padrão de formação em $\mathrm{kJ} / \mathrm{mol}$ e $\mathrm{kcal} / \mathrm{mol}$ de cada substância envolvida na reação, além de muitos outros dados. Portanto, pode-se chegar a entalpia de reação por meio da equação da variação de entalpia (equação 8). As reações estudadas utilizando os métodos AM1, RM1, PM6 e PM7 têm seus resultados apresentados nas Tabelas 1 a 5 a seguir, assim como o respectivo valor experimental de cada entalpia de formação e reação.

Tabela 1. Entalpias de formação $\left(\Delta H_{f}\right)$ e de reação $\left(\Delta H_{r}\right)$ para a combustão do metano. Valores em $\mathrm{kJ} / \mathrm{mol}$.

\begin{tabular}{|c|c|c|c|c|c|c|}
\hline$\Delta \mathbf{H} f$ & $\mathrm{CH}_{4}$ & $2 \mathrm{O}_{2}$ & $\rightarrow$ & $\mathrm{CO}_{2}$ & $2 \mathrm{H}_{2} \mathrm{O}$ & $\Delta \mathbf{H r}=\Sigma \mathbf{H} f_{\mathrm{P}}-\Sigma \mathbf{H} f_{\mathrm{R}}$ \\
\hline AM1 & $-36,73$ & $-113,26$ & & $-334,13$ & $-247,90$ & $\Delta \mathrm{Hr}_{\mathrm{aml}}=-566,68$ \\
\hline RM1 & $-58,07$ & $-86,60$ & & $-341,20$ & $-242,04$ & $\Delta \mathrm{Hr}_{\mathrm{rml}}=-594,00$ \\
\hline PM6 & $-51,37$ & $-70,08$ & & $-354,84$ & $-227,19$ & $\Delta \mathrm{Hr}_{\mathrm{pm} 6=}=-617,68$ \\
\hline PM7 & $-60,24$ & $-35,52$ & & $-353,58$ & $-241,79$ & $\Delta \mathrm{Hr}_{\mathrm{pm} 7}=-705,88$ \\
\hline EXP & $-74,80$ & 0 & & $-393,50$ & $-241,75$ & $\Delta \mathrm{Hr}_{\mathrm{exp}}=-802,19$ \\
\hline
\end{tabular}

Tabela 2. Entalpias de formação $\left(\Delta H_{f}\right)$ e de reação $\left(\Delta H_{r}\right)$ para a combustão do propano. Valores em $\mathrm{kJ} / \mathrm{mol}$.

\begin{tabular}{|c|c|c|c|c|c|c|}
\hline$\Delta \mathbf{H} f$ & $\mathbf{C}_{3} \mathbf{H}_{8}$ & $5 \mathrm{O}_{2}$ & $\rightarrow$ & $3 \mathrm{CO}_{2}$ & $4 \mathrm{H}_{2} \mathrm{O}$ & $\Delta \mathbf{H r}=\Sigma \mathbf{H} f_{\mathbf{P}}-\Sigma \mathbf{H} f_{\mathbf{R}}$ \\
\hline AM1 & $-101,67$ & $-113,26$ & & $-334,13$ & $-247,90$ & $\Delta \mathrm{Hr}_{\mathrm{aml}}=-1326,03$ \\
\hline RM1 & $-94,55$ & $-86,60$ & & $-341,20$ & $-242,04$ & $\Delta \mathrm{Hr}_{\mathrm{rml}}=-1464,19$ \\
\hline PM6 & $-87,69$ & $-70,08$ & & $-354,84$ & $-227,19$ & $\Delta \mathrm{Hr}_{\mathrm{pm} 6=}=1535,19$ \\
\hline PM7 & $-96,56$ & $-35,52$ & & $-353,58$ & $-241,79$ & $\Delta \mathrm{Hr}_{\mathrm{pm} 7=}-1753,76$ \\
\hline EXP & $-103,84$ & 0 & & $-393,50$ & $-241,75$ & $\Delta H r_{\text {exp }}=-2043,67$ \\
\hline
\end{tabular}

Tabela 3. Entalpias de formação $\left(\Delta H_{f}\right)$ e de reação $\left(\Delta H_{r}\right)$ para a combustão do butano. Valores em $\mathrm{kJ} / \mathrm{mol}$.

\begin{tabular}{cccccc|c}
\hline $\mathbf{\Delta} \mathbf{H} f$ & $\mathbf{C}_{\mathbf{4}} \mathbf{H}_{\mathbf{1 0}}$ & $+\mathbf{1 3 / 2 \mathbf { O } _ { \mathbf { 2 } }}$ & $\rightarrow$ & $\mathbf{4} \mathbf{C O}_{\mathbf{2}}$ & $+\mathbf{5} \mathbf{H}_{\mathbf{2}} \mathbf{O}$ & \multicolumn{1}{|c}{$\mathbf{H r}=\mathbf{\Sigma} \mathbf{H} f_{\mathbf{P}}-\mathbf{\Sigma} \mathbf{H} f_{\mathbf{R}}$} \\
\hline AM1 & $-130,46$ & $-113,26$ & & $-334,13$ & $-247,90$ & $\Delta \mathrm{Hr}_{\mathrm{aml}=-1709,45}$
\end{tabular}




\begin{tabular}{lcccc|l} 
RM1 & $-115,64$ & $-86,60$ & $-341,20$ & $-242,04$ & $\Delta \mathrm{Hr}_{\mathrm{rm}=-1896,48}$ \\
PM6 & $-108,92$ & $-70,08$ & $-354,84$ & $-227,19$ & $\Delta \mathrm{Hr}_{\mathrm{pm}=-1991,16}$ \\
PM7 & $-118,03$ & $-35,52$ & $-353,58$ & $-241,79$ & $\Delta \mathrm{Hr}_{\mathrm{pm} 7=-2274,42}$ \\
EXP & $-126,14$ & 0 & $-393,50$ & $-241,75$ & $\Delta \mathrm{Hr}_{\text {exp }=-2656,63}$ \\
\hline
\end{tabular}

Tabela 4. Entalpias de formação $\left(\Delta H_{f}\right)$ e de reação $\left(\Delta H_{r}\right)$ para a combustão do etanol. Valores em $\mathrm{kJ} / \mathrm{mol}$.

\begin{tabular}{|c|c|c|c|c|c|c|}
\hline$\Delta \mathbf{H} f$ & $\mathrm{CH}_{3} \mathrm{CH}_{2} \mathrm{OH}$ & $+3 \mathrm{O}_{2}$ & $\rightarrow$ & $2 \mathrm{CO}_{2}$ & $3 \mathrm{H}_{2} \mathrm{O}$ & $\Delta \mathbf{H r}=\Sigma \mathbf{H} f_{\mathrm{P}}-\Sigma \mathbf{H} f_{\mathrm{R}}$ \\
\hline AM1 & $-262,33$ & $-113,26$ & & $-334,13$ & $-247,90$ & $\Delta \mathrm{Hr}_{\mathrm{aml}}=-809,85$ \\
\hline RM1 & $-233,46$ & $-86,60$ & & $-341,20$ & $-242,04$ & $\Delta \mathrm{Hr}_{\mathrm{rml}}=-915,25$ \\
\hline PM6 & $-229,61$ & $-70,08$ & & $-354,84$ & $-227,19$ & $\Delta \mathrm{Hr}_{\mathrm{pm} 6}=-951,39$ \\
\hline PM7 & $-233,76$ & $-35,52$ & & $-353,58$ & $-241,79$ & $\Delta \mathrm{Hr}_{\mathrm{pm} 7=}-1092,23$ \\
\hline EXP & $-235,09$ & 0 & & $-393,50$ & $-241,75$ & $\Delta \mathrm{Hr}_{\mathrm{exp}}=-1277,16$ \\
\hline
\end{tabular}

Tabela 5. Entalpias de formação $\left(\Delta H_{f}\right)$ e de reação $\left(\Delta H_{r}\right)$ para a combustão do metanol. Valores em

\begin{tabular}{cccccc|c}
\hline $\mathbf{\Delta H} \boldsymbol{f}$ & $\mathbf{C H}_{\mathbf{3}} \mathbf{O H}$ & $\mathbf{3 / 2 \mathbf { O } _ { \mathbf { 2 } }}$ & $\rightarrow$ & $\mathbf{C O}_{\mathbf{2}}$ & $+\mathbf{2} \mathbf{H}_{\mathbf{2}} \mathbf{O}$ & $\Delta \mathbf{H r}=\mathbf{\Sigma} \mathbf{H} \boldsymbol{f}_{\mathbf{P}}-\mathbf{\Sigma} \mathbf{H} \boldsymbol{f}_{\mathbf{R}}$ \\
\hline AM1 & $-238,69$ & $-113,26$ & $-334,13$ & $-247,90$ & $\Delta \mathrm{Hr}_{\mathrm{am} 1=}-421,37$ \\
RM1 & $-209,70$ & $-86,60$ & $-341,20$ & $-242,04$ & $\Delta \mathrm{Hr}_{\mathrm{rm} 1=-485,67}$ \\
PM6 & $-202,25$ & $-70,08$ & $-354,84$ & $-227,19$ & $\Delta \mathrm{Hr}_{\mathrm{pm} 6=-501,87}$ \\
PM7 & $-204,72$ & $-35,52$ & $-353,58$ & $-241,79$ & $\Delta \mathrm{Hr}_{\mathrm{pm} 7=-579,19}$ \\
EXP & $-200,62$ & 0 & $-393,50$ & $-241,75$ & $\Delta \mathrm{Hr}_{\mathrm{exp}}=-676,38$ \\
\hline
\end{tabular}

Uma correlação de aproximação linear entre a entalpia padrão de formação (calculadas no programa MOPAC2012) de todos os compostos envolvidos nas reações selecionadas e as entalpias experimentais, está expressa nos gráficos 1 a 4 a seguir, para cada método separadamente. 
AM1

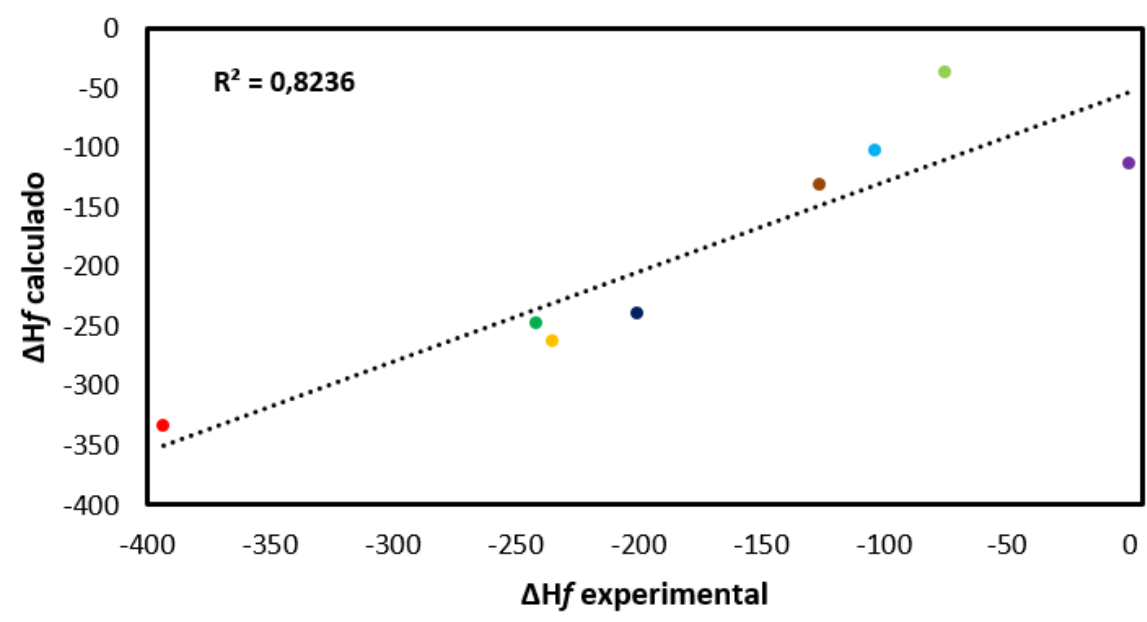

- $\mathrm{CO}_{2}$

$\cdot \mathrm{H}_{2} \mathrm{O}$

$\mathrm{CH}_{3} \mathrm{CH}_{2} \mathrm{OH}$

- $\mathrm{CH}_{3} \mathrm{OH}$

- $\mathrm{C}_{4} \mathrm{H}_{10}$

- $\mathrm{C}_{3} \mathrm{H}_{8}$

- $\mathrm{CH}_{4}$

$\cdot \mathrm{O}_{2}$

Gráfico 1. Correlação linear entre a entalpia padrão de formação dos compostos envolvidos nas reações selecionadas e suas entalpias experimentais, para o método AM1. Valores em kJ/mol.

\section{RM1}

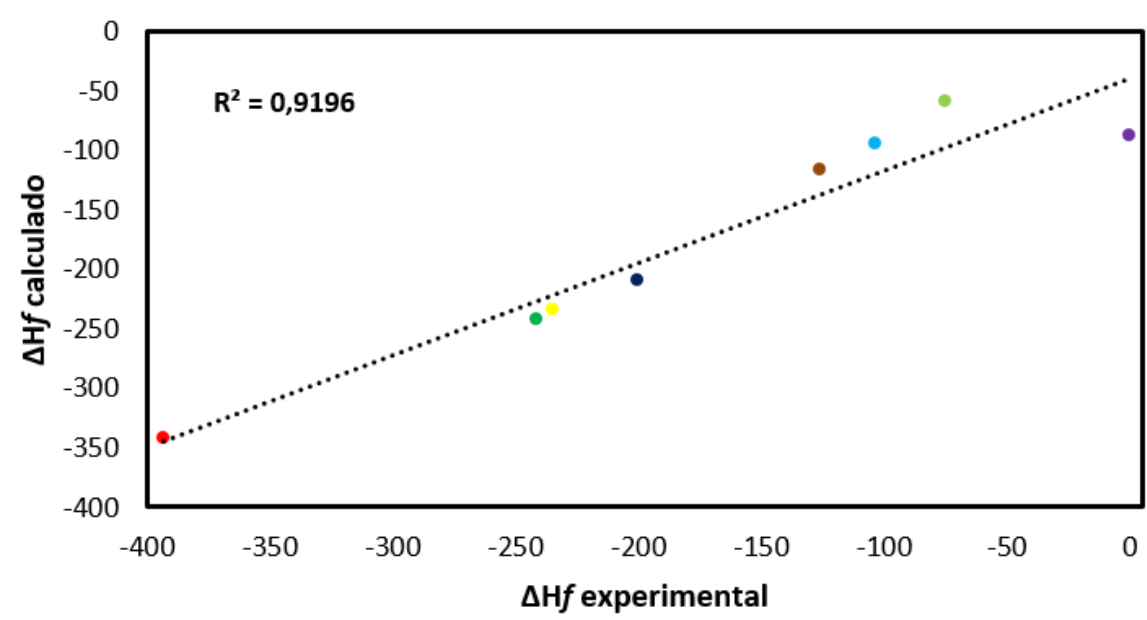

- $\mathrm{CO}_{2}$

$\cdot \mathrm{H}_{2} \mathrm{O}$

$\mathrm{CH}_{3} \mathrm{CH}_{2} \mathrm{OH}$

$\cdot \mathrm{CH}_{3} \mathrm{OH}$

- $\mathrm{C}_{4} \mathrm{H}_{10}$

- $\mathrm{C}_{3} \mathrm{H}_{8}$

- $\mathrm{CH}_{4}$

- $\mathrm{O}_{2}$

Gráfico 2. Correlação linear entre a entalpia padrão de formação dos compostos envolvidos nas reações selecionadas e suas entalpias experimentais, para o método RM1. Valores em kJ/mol. 


\section{PM6}

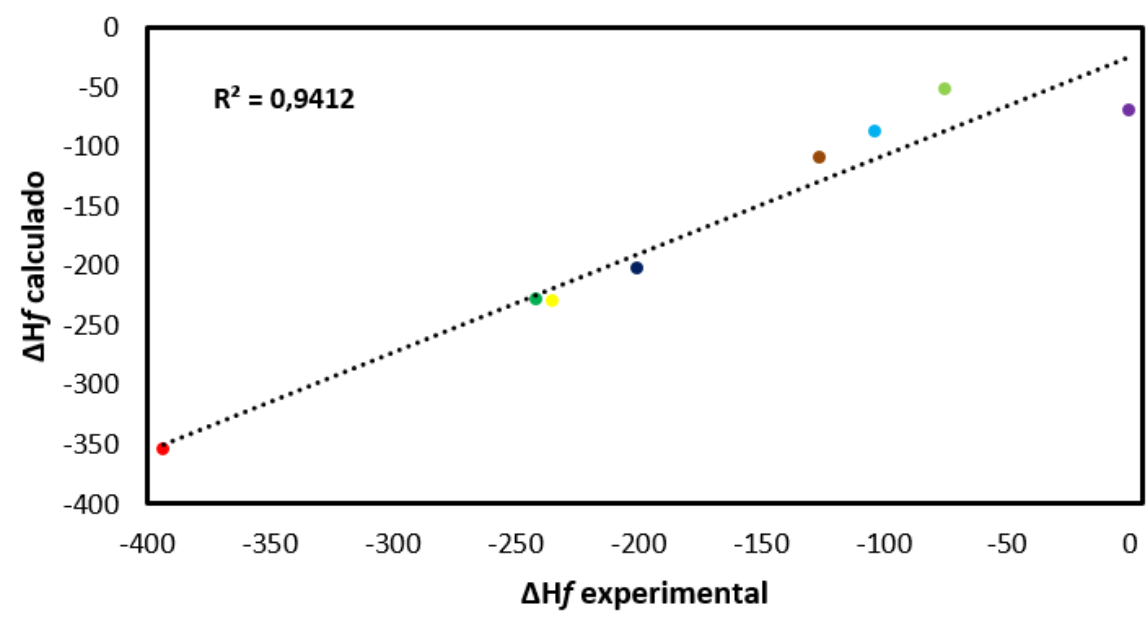

$$
\begin{aligned}
& \cdot \mathrm{CO}_{2} \\
& \cdot \mathrm{H}_{2} \mathrm{O} \\
& \cdot \mathrm{CH}_{3} \mathrm{CH}_{2} \mathrm{OH} \\
& \cdot \mathrm{CH}_{3} \mathrm{OH} \\
& \cdot \mathrm{C}_{4} \mathrm{H}_{10} \\
& \cdot \mathrm{C}_{3} \mathrm{H}_{8} \\
& \mathrm{CH}_{4} \\
& \cdot \mathrm{O}_{2}
\end{aligned}
$$

Gráfico 3. Correlação linear entre a entalpia padrão de formação dos compostos envolvidos nas reações selecionadas e suas entalpias experimentais, para o método PM6. Valores em kJ/mol.
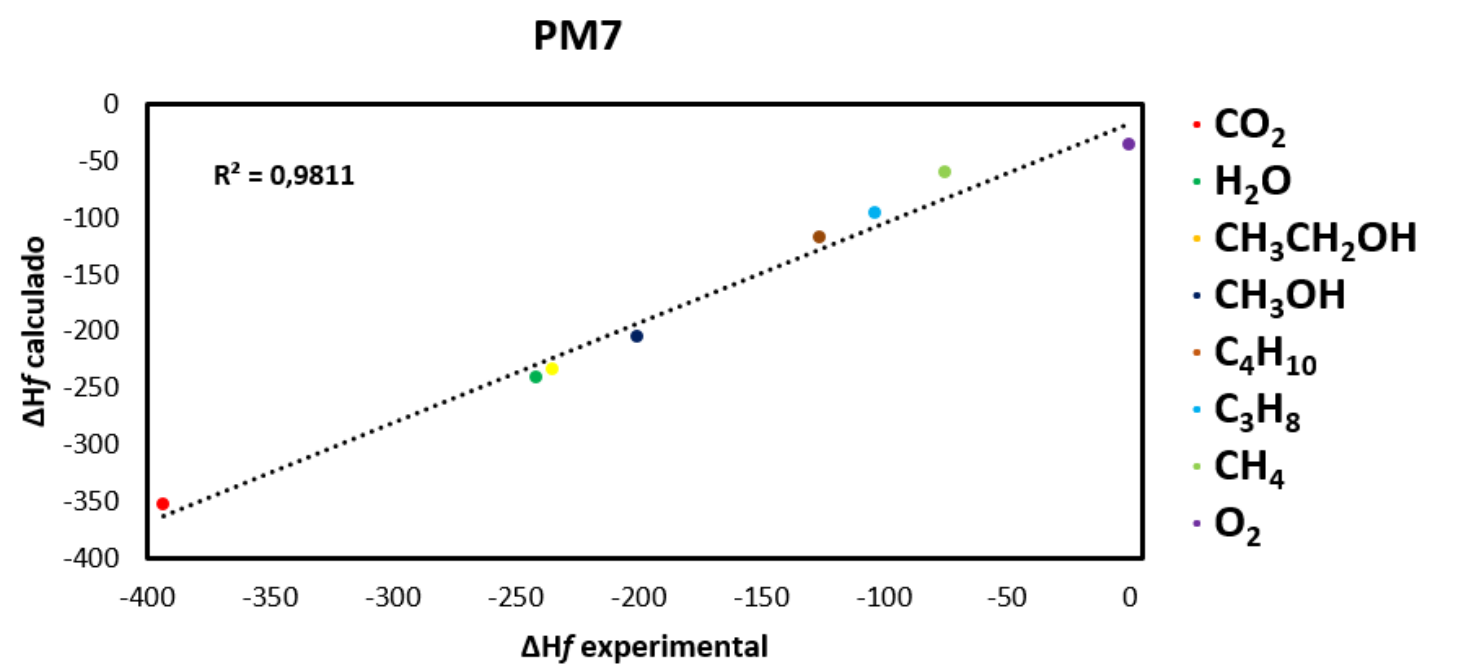

Gráfico 4. Correlação linear entre a entalpia padrão de formação dos compostos envolvidos nas reações selecionadas e suas entalpias experimentais, para o método PM7. Valores em kJ/mol.

Os gráficos acima mostram que dentre os métodos semiempíricos utilizados neste trabalho, as entalpias de formação dos componentes das reações estudadas foram melhor calculadas pelo método PM7, que por sinal é o método parametrizado mais recentemente [26].

Em relação ao tempo computacional, o arquivo de saída do MOPAC2012 ainda fornece o tempo real utilizado para realizar o cálculo, transcorrido desde o início até o fím do processo. Neste trabalho, o tempo médio gasto para o cálculo da entalpia de cada substância foi de 0.8 segundos para o cálculo de cada substância que precisou ser calculada. Uma característica que agrada a dinâmica educacional pelo fato de pode ser feita em pouco tempo. $\mathrm{O}$ fato de vários parâmetros que seriam calculados em um método $a b$ initio já terem sido previamente determinados, faz com que os métodos semiempíricos se tornem muito mais rápidos.

A escolha das reações envolvidas no processo necessita atenção. Como os cálculos de entalpia realizados no MOPAC foram para sistemas gasosos, o valor de referência determinado experimentalmente para a entalpia de formação de todas as substâncias foi à entalpia do seu estado gasoso, dessa forma, puderam ser comparadas no mesmo aspecto. 
Nota-se que nos métodos utilizados, apesar de não exatamente exatos, conseguem se aproximar das entalpias obtidas experimentalmente, e assim, satisfazer a metodologia. Essa aproximação razoável se deve ao fato de os métodos semiempíricos aplicados nos cálculos desse trabalho negligenciarem algumas integrais das equações da teoria quântica, mas que, no entanto, apresentam ótimos resultados relativos.

Outro fator determinante no desvio dos resultados teóricos em relação as entalpias de reação experimentais é que nos cálculos computacionais do MOPAC2012, os resultados para a molécula de oxigênio, possuem a entalpia de formação calculada mais distante do valor experimental que é igual a zero. Tendo em mãos os resultados, pode-se perceber que o método semiempírico que mais se aproximou dos valores experimentais para cada reação foi o PM7.

\subsection{Avaliação das práticas realizadas pelos graduandos}

A partir das respostas dos questionários aplicados aos graduandos, preparou-se a aula teórica de forma a complementar os conhecimentos preestabelecidos dos mesmos. Neste ponto, é importante ressaltar que a grande maioria dos alunos eram capazes de definir entalpia e resolver exemplos simples. No entanto, $25 \%$ dos alunos não foram capazes de relacionar à exemplos práticos do cotidiano, enquanto que, $90 \%$ da turma não sabia como este conteúdo poderia ser trabalhado a partir de um software de computador.

Neste contexto, durante a aula foi apresentado uma breve revisão de métodos teóricos em química, e então eles puderam realizar todas as etapas dos cálculos presentes no roteiro, ao mesmo tempo em que tiravam as dúvidas necessárias. O roteiro de atividades desenvolvido em sala continha as mesmas reações apresentadas no tópico anterior (ver item 3.1). Sendo assim, os alunos conseguiram chegar aos resultados esperados e puderam fazer as comparações apresentadas.

Após o término das atividades, os alunos relataram a sua visão sobre o método que tinham executado, destacando pontos positivos e negativos, assim como discutindo a viabilidade deste método ser inserido como metodologia ao ensino.

Os graduandos citaram muitos aspectos em comum, tanto positivos quanto negativos, que podem ser vistos na Tabela 6 , juntamente com alguns pontos observados pelos autores:

Tabela 6. Pontos positivos e negativos apontados pelos graduandos e observados após a aplicação do método no MOPAC2012.

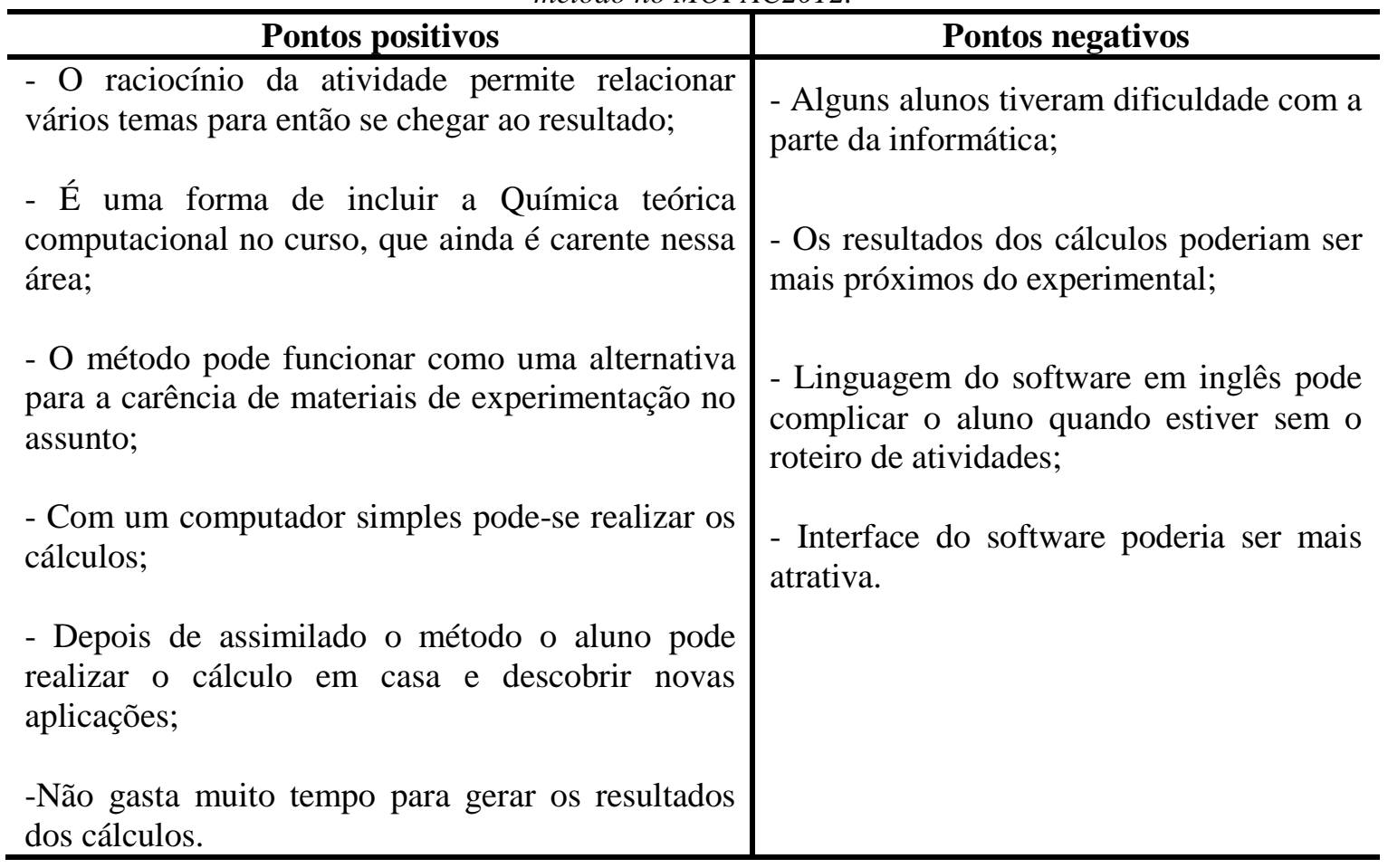


Os alunos ainda relataram que o método sendo bem trabalhado e um pouco melhorado, poderá ser um relevante meio de ensino não só nos tópicos da termodinâmica química como em outros assuntos relacionados.

\section{CONCLUSÃO}

Com os resultados obtidos neste trabalho, acredita-se que essa metodologia apresenta condições de ser uma alternativa para o ensino de entalpia de reação, podendo ser aplicada a cursos de graduação em Química, desde que seus recursos sejam aplicados no momento correto e com acompanhamento teórico adequado, para que os alunos relacionem da melhor maneira possível a prática ao conteúdo que pretende ser trabalhado. Dessa forma, com esta proposta espera-se diversificar os métodos de ensino da entalpia, ampliando a motivação dos alunos pelo tema. Apesar de os resultados apresentarem um certo desvio dos valores experimentais, observa-se que a praticidade da aplicação destes métodos é um ponto positivo na inserção como recurso didático, uma vez que computadores pessoais podem ser utilizados obtendo-se resultados em um curto intervalo de tempo.

\section{REFERÊNCIAS BIBLIOGRÁFICAS}

1. Nunes AS, Adorni DS. O ensino de química nas escolas da rede pública de ensino fundamental e médio do município de Itapetinga-BA: O olhar dos alunos. In: Anais Encontro Dialógico Transdisciplinar Enditrans, Vitória da Conquista-BA; 2010.

2. Lopes ARC. Conhecimento escolar: Ciência e cotidiano. Rio de Janeiro: Ed.uerj; 1999.

3. Rodello IA, Sakai RK, Manoel EF. Um Ambiente Virtual para Auxiliar o ensino de Química em escolas de Ensino Fundamental. Anais do VIII WIE; 2002.

4. Ribeiro AA, Greca IM. Simulações Computacionais e ferramentas de modelização em educação química: uma revisão da literatura publicada. Química Nova. 2003; 26(4):542-549, doi: 10.1590/S0100-40422003000400017.

5. Covolan SCT, Silva DA. Entropia no ensino médio: utilizando concepções prévias dos estudantes e aspectos da evolução do conceito. Ciência \& Educação. 2005 11(1):97-117, doi: 10.1590/S151673132005000100009

6. Grings ETO, Caballero C, Moreira MA. Avanços e retrocessos dos alunos no campo conceitual da Termodinâmica. Revista Electrónica de Enseñanza de las Ciencias. 2008 7(1):23-46.

7. Köhnlein JFK, Peduzzi SS. Um estudo a respeito das concepções alternativas sobre calor e temperatura. Revista Brasileira de pesquisa em Educação em Ciências. 2002 2(3):25-35.

8. Silva JLPB. Porque não estudar entalpia no ensino médio. QNEsc. 2005 22:22-25.

9. MOPAC2012, Stewart JJP, Stewart Computational Chemistry, Colorado Springs, CO, USA, HTTP://OpenMOPAC.net (2012).

10. Pádua AB, Pádua CG, Silva JLC, Martins RS, Postali FB, Tiritan LAC. Termodinâmica clássica ou termodinâmica do equilíbrio: aspectos conceituais básicos. Londrina, Semina: Ciências Exatas e Tecnológicas. 2008; 29(1): 57-84. doi: 10.5433/1679-0375.2008v29n1p57.

11. Baldow R, Monteiro Jr FN. Os livros didáticos de física e suas omissões e distorções na história do desenvolvimento da Termodinâmica. ALEXANDRIA: Revista de Educação em Ciência e Tecnologia. 2010; 3(1): 3-19. doi: 10.5007/38013.

12. Pádua AB, De Pádua CG, Silva JLC. A História da Termodinâmica Clássica: uma Ciência Fundamental. Londrina: Eduel; 2009. 134p.

13. Barros HLC. Processos endotérmicos e exotérmicos: uma visão atômico-molecular. Química Nova na Escola. 2009; 31(4) 241-245.

14. Jacques V, Alves Filho JP. O conceito de energia: os livros didáticos e as concepções alternativas. XI Encontro de Pesquisa em Ensino de Física, Curitiba; 2008.

15. Atkins P, Jones L. Princípios de química: questionando a vida moderna e o meio ambiente. Tradução Ricardo Bicca de Alencastro. 3a. ed., Porto Alegre: Bookman; 2006.

16. Lambert FL. Configurational entropy revisited. J. Chem. Educ. 2007; 84: 1548-1550.

17. Atkins P, de Paula J. Physical Chemistry. 8a. ed. Oxford University Great Britain; 2006.

18. STEWART JJP. Optimization of parameters for semiempirical methods VI: more modifications to the NDDO approximations and re-optimization of parameters. 2013; 19(1): 1-32. 
19. Lewars EG. Computational Chemistry: Introduction to the Theory and Applications of Molecular and Quantum Mechanics 2a. ed. New York: Springer; 2011.

20 Trsic M, Pinto MFS. Química Quântica: Fundamentos e Aplicações. Manole, Barueri - 2009.

21. Atkins P, de Paula J, Friedman R. Quanta, Matter, and Change: A molecular approach to physical chemistry. 2a. ed. New York: W. H. Freeman and Company; 2009.

22. Dewar MJS, Zoebisch EG, Healy EF, Stewart JJP. Development and use of quantum mechanical molecular models. 76. AM1: a new general purpose quantum mechanical molecular model. J Am Chem Soc. 1985 June;107(13):3902-3909 DOI: 10.1021/ja00299a024

23. Galvão DS, Soos ZG, Ramasesha S, Etemad S. A parametric method 3 (PM3) study of trans-stilbene. The Journal of Chemical Physics 1993; 98: 3016. Doi: 10.1063/1.464128

24. Rocha GB, Freire RO, Simas A, Stewart JJPJ. RM1: a reparameterization of AM1 for H, C, N, O, P, S, F, Cl, Br, and I. J. Comput. Chem. 2006; 27(10): 1101-1111. DOI: 10.1002/jcc.20425

25. Stewart, J.J.P. Optimization for semiempirical methods V: Modification of NDDO approximations and application to 70 elements. Journal of Molecular Modeling. 2007; 13(12): 1173-1213. doi: 10. 1007 / s00894-007-0233-4

26. Stewart JJP. Optimization of parameters for semiempirical methods VI: Modification of NDDO approximations and re-optimization of parameters. Journal of Molecular Modeling. 2013; 19(1): 1-32. doi:10.1007/s00894-012-1667-x

27. Valente JA. Formação de educadores para o uso da informática na escola. Campinas- SP: Unicamp/Nie; 2003.

28. Chaves EOC. Tecnologia e Educação: o futuro da escola na sociedade da Informação. Campinas: Mindware Editora, 1998.

29. Taylor RP. The Computer in the School: Tutor, Tool, Tutee. Cite journal. 1980; 3(2): 1-10.

30. Rodrigues C, Diniz J. Ambiente virtual: ainda uma proposta para o ensino. Ciências \& Cognição. 2008; 13(2): 71-83.

31. Araujo, I. S.; Veit, E. A. Modelagem computacional no Ensino de Física. Revista do Centro de Educação da Universidade Federal de Alagoas. CEDU-n; 2005.

32. Raupp D, Serrano A, Martins TLC. A evolução da química computacional e sua contribuição para a educação em Química. Revista Liberato. 2008; 9(12):13-22.

33. Ramos AF, Serrano A. Modelagem molecular no Ensino de Ciências: uma revisão da literatura no período 2001-2011 acerca da sua aplicabilidade em atividades de ensino. Acta Scientiae. 2013; 15(2):363-382. 